\title{
The problem of recovery of the patients with cerebrovascular accident (CVA): the capabilities of psychological techniques in the improvement of cognitive functions
}

\author{
Irina Skirtach ${ }^{1,2, *}$ and Evgenia Pokul $^{1}$ \\ ${ }^{1}$ Don State Technical University, 344003, Gagarin Sq., 1, Rostov-on-Don, Russia \\ ${ }^{2}$ Southern Federal University, 344006, Bolshaya Sadovaya str., 105/42, Rostov-on-Don, Russia
}

\begin{abstract}
If the appropriate combined measures are not taken to treat the manifestations of cognitive disorders, they have a tendency to progress towards worsening over time in the patients with CVA. The most common cognitive dysfunctions are as follows: disorders of attention, thinking, memory and executive functions. More and more studies are focused on the application of the various methods and techniques for neurorehabilitation of these disorders. Evaluation of the effect of adding the neurorehabilitation techniques in the treatment program in the early recovery period was a part of the objectives of this study. The sample consisted of 70 people with CVA. The study was carried out in the hospital environment. During 10 days, the standard scheme of the drug treatment was supplemented with the neurorehabilitation method using the "Brain jogging" technique and group work techniques in the experimental group. A battery of the psychodiagnostic tests was used, which included the "MMSE Scale" test, PieronRuser test, Ebbinghaus test, and "10-word memorization" technique (according to A.R. Luria). Based on the study results, the features of the dynamics of recovery in the patients with CVA of indicators of cognitive functions are described due to the inclusion of psycho-gymnastic techniques in the rehabilitation program.
\end{abstract}

\section{Introduction}

All over the world, stroke is currently considered one of the most serious and common cerebrovascular diseases. Being one of the leading causes of death in the advanced industrial countries, stroke increasingly affects the young and middle-aged people resulting to their disability [1-2]. Even in the case of favorable treatment outcome for the majority of patients, full recovery and return to their usual lifestyle and work represent a serious challenge. As a result, such social institutions as the family and even the state are put at risk. Thus, the issues of rehabilitation of these patients are acute medical and psychological and social ones in nature.

\footnotetext{
${ }^{1}$ Corresponding author: limpopo-is@yandex.ru
} 
A stroke is a cerebral circulation disorder, in any part of the brain where blood flow decreases or stops.

The most common disorders after a stroke are motor disturbances in the form of paralyses and pareses of varying manifestation degrees, as well as cognitive disorders [3]. Memory and intelligence, attention and mental capacity, affection and volition, related to the brain integrative functions, are often affected by focal brain lesions [4- 6]. Cognitive impairments occur in the overwhelming number of CVA patients and adversely impact the personal, social and occupational adaptation like motor deficit.

Post-stroke cognitive impairments worsen the prognosis, increase the mortality rate and stroke recurrence by 3 times, an increase in the post-stroke functional impairments is observed that makes rehabilitation difficult.

Rehabilitation is based on the neuroplasticity - the brain property to change its functional and structural reorganization, the capability of its different structures to participate in various activities [7]. It is stated that even at a mature age, the brain retains high neuroplasticity and learning capability due to the modulation of synaptic transmission and synaptogenesis; this provides great capabilities for the development of neurorehabilitation programs for both young and elderly patients [8-9].

Currently, the post-stroke period is usually divided into 4 periods [10]: acute period; early recovery period; late recovery period; residual period.

There is a whole range of medical and psychological principles for the formation of neuropsychological rehabilitation measures, among which the early onset, consistency and stage-by-stage approach to the implemented measures are of particular importance [11].

Neurorehabilitation is one of the relatively new directions of rehabilitation. This is a progressive, dynamic and focused process which goal consists in achieving the optimal level of physical, cognitive, emotional, social, communicative and functional activity by the patient with the disorders occurred [12].

Xiao-Di Xu's study, which focuses on the approaches in the field of neuropsychological rehabilitation, noted that the delay in the correction of attention and cognitive function inevitably results in the progression of the deficit of the above functions in the patients during the first year after a stroke. Failure of voluntary attention will interfere with the restoration of long-term memory [13]. However, an analysis of the advanced experience indicates the most researchers to use a psychological rehabilitation only during the late recovery and residual periods noting the high efficiency of rehabilitation measures directed at psychological development; the main attention is focused on the motor programs that stimulate the neuronal activity in the brain [14-16]. The experience of the Chinese scientists in combating the consequences of stroke allows to note the increased focus on the inclusion of nontraditional techniques combined with various drugs in the rehabilitation program. For example, in the studies focused on the possibilities of using Chinese traditional medicine, the data were obtained on a considerable reduction in muscle spasticity in CVA patients due to the use of therapeutic physical training techniques [17], Chinese massage therapy, [18] and head acupuncture [19].

The "Brain jogging" technique is one of the neurorehabilitation methods. According to a number of studies, the use of cognitive exercises has a positive effect on the recovery process of patients with diseases accompanied by impaired local functions of the brain, such as chronic cerebrovascular disease, mental debility, mild cognitive dysfunction, etc. [20, 21$]$. In addition, the use of this set of exercises showed better results in solving the problem of improving the performance of children with special educational needs [22], and also, being included in the health promotion systems in kindergartens and schools, proved to be an effective measure to activate the mental activity of children and teenagers [23]. There are separate works focused on the use of cognitive techniques by the patients after ischemic stroke in order to correct some cognitive functions [22]. 
The issue of using this technique in a complex of rehabilitation measures for CVA patients in the early recovery period became the subject of the study described in this paper. An additional, statistically valid evidence of the effect of cognitive exercises on the dynamics of recovery of higher mental functions is required.

\section{Study procedure and methods}

Enrolled set consisted of 70 people (experimental group - the patients of the Acute Cerebrovascular Disorder Department of the Rostov Regional Clinical Hospital, control group the patients of the Hospital of the Rostov State Medical University, the Neurological Department for patients with Acute Cerebrovascular Disorders): 39 women, 31 men, aged 3975 years $(M=54.7, S D=10.6)$ that corresponds to the middle and old age according to WHO age grouping of population.

All subjects were right-handers. All subjects had marked symptoms of cognitive disorder, which included: deficit of attention and attention concentration, defective memory, slow thinking, fatigue during intense mental work due to hemorrhagic and ischemic stroke, and they were in a hospital at the first stage of recovery at the study entry.

The control and experimental groups were equal in the number of subjects and did not significantly differ in age composition. For the latters, during ten days the standard drug treatment regimen was supplemented with a rehabilitation program using "Brain jogging" exercises and group work techniques. The technique of performing "Brain jogging" exercises is based on the movements realized crosswise by a person. This motor skill activates the system of step-by-step synchronization of the left and right hemispheres, which, due to the optimal distribution of stress between the cerebral cortex and the subcortical structures of the left and right hemispheres, ensures plastic restructuring of brain neurons and their further well-balanced work. The classes were held before midday, when the subjects are most efficient. Compliance with usual daily routine was an important condition for the study. The patient should know when the measures to improve his/her health would be taken that helped him/her feel confident and safe. Rehabilitation activities containing a set of "Brain jogging" exercises were carried out taking into account the fact that group activities provide greater involvement of each group member in the rehabilitation process. The help to the patient should be also provided in a gentle form that ensured the preservation of selfrespect as too obvious help or pressure by the investigator could cause negative emotions in the patient associated with the unwillingness to demonstrate the failures that occur during the recovery process to the rest of the group. During the study, mutual assistance between the participants was observed in each microgroup. More cognitive safe patients explained in a verbal and non-verbal way to others the instructions they did not understand. After the group activities, the forms with "Brain jogging" exercises were given to the patients for independent study to be performed in the formed small groups. At the baseline and at the end of the experiment, both samples were tested to study the dynamics of a number of indicators: short-term memory, attention concentration and stability, cognitive impairments, and the associative thinking speed.

The study methods include: experimental method, scaling, statistical methods. The methodological toolkit included the following methods: "MMSE (Mini-Mental State Examination) Scale" test, Pieron-Ruser test, Ebbinghaus test, and "10-word memorizing" technique (according to A.R. Luria).

The goal of the study was to study the cognitive function dynamic pattern in CVA patients when using the set of "Brain jogging" exercises at the initial stage of rehabilitation.

The following assumptions were analyzed: 1) the cognitive function dynamic pattern differs in CVA patients when the adapted set of "Brain jogging" exercises is included in the rehabilitation program and the patients for whom brain jogging was not included in the re- 
habilitation program; 2) during the rehabilitation, the use of the set of "Brain jogging" exercises has relation to the dynamic pattern in the indicators of association productivity, level of attention concentration, short-term memory, cognitive status.

\section{Discussion}

Data of the psychodiagnostic study were analyzed using the methods of mathematical statistics. For the experimental group, it was found that a comparison by the medians of MMSE technique indicators of the first and second tests demonstrated a positive dynamics of the cognitive sphere indicators from 25 to 29 scores by the following parameters: spatial and temporal orientation, perception, capability to write, read, and count. According to these indicators, a positive increase from 25 to 27 scores was also found in the control group. Based on Wilcoxon rank sum test, the statistical analysis demonstrated the significant differences between the first and second psychodiagnostic studies of the cognitive sphere indicators in both control $(\mathrm{z}=4.457345, \mathrm{p}$-value $=0.000008)$ and experimental $(\mathrm{z}=4.859943$, $\mathrm{p}$-value $=0.000001)$ groups. When comparing the data obtained in the study of Gavrilova T. A., who used the "Brain jogging" technique in the rehabilitation of patients with chronic cerebrovascular disease and mild cognitive dysfunction at the sanatorium treatment stage, the parameter dynamics by MMSE scale was 0.7 points [21] that can be regarded as lower indicator compared to the results obtained in our study when the set of "Brain jogging" exercises was included at the initial stage of the patient rehabilitation. The data of Grigoriev D.N. confirm the rationale for using this technique for persons who have difficulties in process of training and skills development [24].

Disorder of concentration and volume of voluntary attention, combined with impaired gross and fine motor skills, is a common consequence of stroke. Pieron-Ruser methodology allows to evaluate the speed and quality of a simple skill development, learning of a new way of action, indicators of volume and stability of voluntary attention, capacity of hand motor skills. At the end of the study, the median indicator of volume and stability of voluntary attention changed from 3 to 4 in the experimental group, from 2 to 3 in the control group. Based on Wilcoxon rank sum test, the statistical analysis demonstrated the significant differences between the first and second psychodiagnostic studies of the indicators of volume and stability of voluntary attention in both control $(\mathrm{z}=4.622599, \mathrm{p}$-value $=$ $0.000004)$ and experimental $(z=3.919930, \mathrm{p}$-value $=0.000089)$ groups. Information on the positive effect of the "Brain jogging" technique in the scientific literature is available only for children with special needs and general speech underdevelopment [25]. Our study showed that this psycho-gymnastics can also be used in the rehabilitation of CVA patients.

Taking into account the fact that the "Brain jogging" technique improves interhemispheric interaction and activates the function of both hemispheres, the decision was taken to evaluate the possibilities of using this technique to restore associative thinking; the right hemisphere is responsible for both associative thinking and processing and generating images and symbols used in the process of associative thinking. In the process of rehabilitation, this thinking becomes a resource that is used during the rehabilitation of CVA patients allowing to build associative chains between what the patient remembers and the rest volume of the blocked memory. We used the Ebbinghaus test to identify the level of performance indicators of associations in CVA patients, and its changes during a given time interval.

The indicator median of the performance of associative thinking was 114 scores before the study, and 57 scores after the study in the experimental group, it was 80 scores before the study and 60 scores after the study in the control group, respectively. Based on Wilcoxon rank sum test, the statistical analysis demonstrated the significant differences between the first and second psychodiagnostic studies of the performance of associative thinking 
indicators in both control $(\mathrm{z}=4.265581, \mathrm{p}$-value $=0.000020)$ and experimental $(\mathrm{z}=$ 5.086213 , p-value $=0.000000)$ groups.

The analysis of changes in the indicators of the memory level for words (auditory), memorizing, retention, reproduction, voluntary attention, voluntary attention by the "10word memorizing" technique (according to A.R. Luria) showed an increase in the indicator value from 4 to 7 scores (which is an increase of 30\%) upon completion of the rehabilitation program with the inclusion of the neurorehabilitation method in the experimental group, and an increase from 4 to 5 scores in 2 tests in the control group. Based on Wilcoxon rank sum test, the statistical analysis demonstrated the significant differences between the first and second psychodiagnostic studies of the memory indicators in both control $(\mathrm{z}=$ 4.703046, $\mathrm{p}$-value $=0.000003)$ and experimental $(\mathrm{z}=5.086213, \mathrm{p}$-value $=0.000000)$ groups. In regard to the patients with chronic cerebrovascular disorders and mild cognitive dysfunction, the data of Gavrilova T.A. also show a positive effect ( 0.5 scores) against these indicators in connection with the use of psycho-gymnastics [21]. In regard to the use of the "Brain jogging" technique in the recovery of memory functions in CVA patients, the data of Bykova N.V. confirm a positive trend: improvement of the memory for images by $11 \%$, and the memory for numbers by $12 \%$ at the sanatorium stage of rehabilitation [23].

Based on Mann-Whitney U test, the subsequent evaluation of the dynamic pattern in the studied parameters between the groups showed that statistically significant differences in the changes that occurred in the control and experimental groups were confirmed in regard to the indicators shift in the volume and stability of voluntary attention, the performance of associative thinking, the memory level for words (auditory), memorizing, retention, reproduction, voluntary attention, emotional state, but no significant differences in regard to the indicators shift in the cognitive sphere were detected according to the MMSE test (Table 1).

Table 1. Comparison of the difference between the results in the control and experimental groups during the primary and repeated measurement of the studied indicators according to the MannWhitney $\mathrm{U}$ test $(\mathrm{p} \leq 0.05)$.

\begin{tabular}{|l|l|l|l|l|l|}
\hline \multicolumn{1}{|c|}{$\begin{array}{c}\text { Psychodiagnostic tech- } \\
\text { niques }\end{array}$} & $\mathbf{U}$ & $\mathbf{Z}$ & $\mathbf{p}$-value & $\mathbf{Z}$ - adjusted & p-value \\
\hline "MMSE Scale" test & 500.5000 & 1.309689 & 0.190302 & 1.326042 & 0.184827 \\
\hline Pieron-Ruser test & 457.5000 & 1.814771 & $0.049560^{*}$ & 1.957781 & 0.050257 \\
\hline Ebbinghaus test & 313.0000 & 3.512082 & $0.000445^{*}$ & 3.519355 & 0.000433 \\
\hline $\begin{array}{l}\text { "10-word memorizing" } \\
\text { technique }\end{array}$ & 420.0000 & 2.255250 & $0.024118^{*}$ & 2.332802 & 0.019659 \\
\hline
\end{tabular}

Notes: ${ }^{*}$ - statistically significant differences

As all other conditions in both groups were equal, it can be concluded that the use of the "Brain jogging" technique in the rehabilitation program had a reliably positive effect on improving the performance indicators of associations, short-term memory of CVA patients.

The obtained results can be explained by the fact that the "Brain jogging" exercises based on stimulation of various brain systems (optical-motor, kinesthetic, auditory, visual) increased the flow of sensory information passing through the nervous system, stimulate the work of superior brain functions (speech, gnosis praxis), thereby enhancing the neuroplasticity properties that affect the level recovery of short-term memory, performance of associations.

As a result of the performed correlation analysis (using the method of Spearmen's rankorder correlation), a connection was found between the presence of the "Brain jogging" technique with a shift in the performance indicators of associations, level of attention concentration, and short-term memory at the initial stage of the rehabilitation program (Table 2). 
Table 2. The results of Spearman correlation analysis of the connection between the use of the "Brain jogging" technique in the rehabilitation program of the subjects with the change in the psychological characteristics under study $(\mathrm{p} \leq 0.05)$.

\begin{tabular}{|c|c|c|c|}
\hline $\begin{array}{c}\text { Psychological characteristics of } \\
\text { the subjects under study }\end{array}$ & $\begin{array}{c}\text { Degree of strength and - } \\
\text { significance of correlation rela- } \\
\text { tionship }\end{array}$ & Spearman R & p-value \\
\hline $\begin{array}{c}\text { Dynamics of "Association perfor- } \\
\text { mance" indicator }\end{array}$ & Fast and highly significant & 0.74 & 0.00015 \\
\hline $\begin{array}{c}\text { Dynamics of "Short-term memory" } \\
\text { indicator }\end{array}$ & Fast and highly significant & 0.72 & 0.01 \\
\hline $\begin{array}{c}\text { Dynamics of "Attention concentra- } \\
\text { tion level" indicator }\end{array}$ & Moderate and significant & 0.51 & 0.0503 \\
\hline $\begin{array}{c}\text { Dynamics of "Cognitive status" } \\
\text { indicator }\end{array}$ & Slow, insignificant & 0.21 & 0.347 \\
\hline
\end{tabular}

Analysis of the data obtained allows to assume that the neurorehabilitation methods used in a certain way affect the change in the psychological characteristics of the subjects. The study findings indicate that the "Brain jogging" technique can be especially effective in relation to impaired performance of associative thinking and short-term memory which are common consequences of stroke.

\section{Summary}

Rehabilitation of the cognitive functions developed in parallel with motor rehabilitation. It includes many specific approaches, many of which have been successfully used in the apoplectic patients, preferably in the residual period. The study findings allow to conclude that the inclusion of psycho-gymnastic "Brain jogging" technique is related to the improvement in the recovery dynamic pattern of such cognitive indicators as the volume and stability of voluntary attention, the performance of associative thinking, the level of memory for words (auditory), memorizing, retention, reproduction in the rehabilitation program of CVA patients. This technique, included at the early stage of rehabilitation, combined with a group form of work, will allow the patients to adapt more quickly and improve quality of their life.

The use of modern methods of cognitive rehabilitation (classes with a neuropsychologist, psycho-gymnastics, group work, neurofeedback training, etc.), especially used in combination, can significantly increase the level of cognitive functions, reduce the risk of complications and intensify the patient's involvement in the rehabilitation process. Further studies in this field should be carried out in order to develop an optimal scheme and duration of the neurorehabilitation course, as well as to take into account the severity of the impaired anatomical formations and physiological processes.

\section{References}

1. D. Sofer, Study assesses the global stroke burden. AJN The American Journal of Nursing, 116(9), 16 (2016) doi: 10.1097/01.NAJ.0000494678.00595.a2

2. S. Satoshi, et al., Low free triiodothyronine predicts poor functional outcome after acute ischemic stroke. Journal of the neurological sciences, 368, 89-93 (2016) doi: 10.1016/j.jns.2016.06.063

3. B. Casolla, et al., Biological and imaging predictors of cognitive impairment after stroke: a systematic review. Journal of neurology 266(11), 2593-2604 (2019) doi: $10.1007 / \mathrm{s} 00415-018-9089-\mathrm{z}$ 
4. S. Clarke, Identifying patterns of cognitive deficits: the path to better outcomes after stroke. Journal of Neurology, Neurosurgery \& Psychiatry, 91(5), 449-45 (2020) doi: 10.1136/jnnp-2019-322429

5. I. Jacomb, et al., Cognitive outcomes of pediatric stroke. Child Neuropsychology, 24(3), 287-303 (2018) doi:10.1080/09297049.2016.1265102

6. M. Triviño, et al., Effectiveness of a neuropsychological treatment for confabulations after brain injury: A clinical trial with theoretical implications. PloS one, 12(3), e0173166 (2017) doi: 10.1371/journal. pone.0173166

7. C. Norise, R. H. Hamilton, Non-invasive brain stimulation in the treatment of poststroke and neurodegenerative aphasia: parallels, differences, and lessons learned, Frontiers in Human Neuroscience, 10, 675 (2017) doi: 10.3389/fnhum.2016.00675

8. I. V. Sidyakina, et al., Modern methods of rehabilitation for the post-stroke patients, S.S. Korsakov Journal of Neurology and Psychiatry, 12, 76-80 (2014) doi: 10.17116/ jnevro201411412272-77

9. G. Berlucchi, Brain plasticity and cognitive neurorehabilitation. Neuropsychological rehabilitation, 21(5), 560-578 (2011) doi: org/10.1080/09602011.2011.573255

10. J. Bernhardt, et al., Agreed definitions and a shared vision for new standards in stroke recovery research: the stroke recovery and rehabilitation roundtable taskforce, International Journal of Stroke, 12(5), 444-450 (2017) doi: $10.1177 / 1747493017711816$

11. P. Langhorne, J. Bernhardt, G. Kwakkel, Stroke rehabilitation. The Lancet, 377(9778), 1693-1702 (2011) doi: 10.1016/S0140-6736(11)60325-5

12. D. Hebert, et al., Canadian stroke best practice recommendations: stroke rehabilitation practice guidelines, update 2015. International Journal of Stroke, 11(4), 459-484 (2016) doi:10.1177/1747493016643553

13. X.-D. Xu, et al., Outcomes of neuropsychological interventions of stroke. Ann. Indian Acad. Neurol, 16(3), 319-328 (2013) doi: 10.4103/0972-2327.116909

14. N. A. Antonova, E. B. Kusnetsova, I. I. Sholomov, Alexithymia in the patients with brain infarction of sinistrocerebral localization in the late recovery period, S.S. Korsakov Journal of Neurology and Psychiatry, Special issues, 115(10), 3-6 (2015) doi: 10.17116/jnevro2015115102?-?

15. E. Fernandez, et al., Effectiveness of a computer-based training program of attention and memory in patients with acquired brain damage, Behavioral Sciences, 8(1), 4 (2018)

16. N. G. Ermakova, Psychological aid to the post-stroke patients with motor impairments and their relatives, International Research Journal, 8(50), Part 4, 43-47 (2016) doi: 10.18454/IRJ.2016.50.154

17. W. Zhu et al., Clinical efficacy and sEMG analysis of a new traditional Chinese medicine therapy in the treatment of spasticity following apoplectic hemiparalysis. Acta Neurologica Belgica, 114(2), 125-129 (2014) doi: 10.1007/s13760-014-0279-x

18. Q. Zeng, et al., Mistakes in application of tuina therapy for stroke rehabilitation. Journal of Acupuncture and Tuina Science, 9(1), $51-54$ (2011) doi: 10.1007/s11726011-0470-z

19. Q. Zhang, et al., Standardization of rehabilitation program for post-apoplectic limb spasm treated by Tongjing Tiaoxing tuina and scalp acupuncture with physical therapy. Medicine, 99(21), e20368 (2020) doi: 10.1097/MD.0000000000020368 
20. V. I. Skvortsova, et al., Results of the implementation of the "Package of measures to improve medical care for patients with acute cerebrovascular disorders in the Russian Federation", S.S. Korsakov Journal of Neurology and Psychiatry, 118(4), 5-12 (2018) doi: 10.17116/jnevro2018118415-12

21. T. A. Gavrilova, et al., Possibility of correction of mild cognitive impairments using the technique of "complex brain jogging" therapeutic gymnastics under the conditions of a sanatorium, Kremlin Medicine Journal, 4, 46-48 (2013)

22. M. Katan, A. Luft, Global burden of stroke, Seminars in neurology, 38(2) (2018) doi: $10.1055 / \mathrm{s}-0038-1649503$

23. N. V. Bykova, Brain jogging in the physical education classes as a method to activate mental activity, Methodist, 4, 56-57 (2017)

24. D. N. Grigoriev, O. O. Zagury, Higher education for people with disabilities: Russia and Israel experience, Scientific almanac, 9-1, 236-239 (2016) doi: 10.17117/na.2016.09.01.236

25. E. V. Gorshkova, Psychological and pedagogical assistance to preschool children using methods of psychotechnics based on movement, Bulletin of practical psychology of education, 17(1), 21-31 (2020) doi:10.17759/bppe.2020170103 